\title{
El sector licorero del Ecuador frente a las medidas tributarias impuestas
}

\section{The liquor sector in Ecuador front of the tax measures imposed on}

Mónica Patricia Larrea Paredes

Universidad Ecotec, Ecuador

María de Lourdes Larrea Paredes

Universidad Tecnológica Equinoccial, Ecuador

Autor para correspondencia: mlarrea@ecotec.edu.ec, llarrea@ute.edu.ec

Fecha de recepción: 09 de agosto de 2018 - Fecha de aceptación: 15 octubre de 2018

Resumen: Esta investigación realiza un análisis del sector licorero el que representa un importante rubro de la economía ecuatoriana, mismo que está integrado por importadores y productores locales que en muchos casos recurren a la importación de bienes para la elaboración de sus productos. A nivel mundial los estados buscan mejorar su recaudación fiscal para la ejecución de obras o mejoramiento de los servicios entregados a la ciudadanía, en el Ecuador con este fin se aplican una serie de tributos como impuesto al valor agregado IVA, impuesto a los consumos especiales ICE, aranceles a la importación, salvaguardias, impuesta a la renta IR, entre otros. El presente estudio se enfocó en el sector importador por la serie de medidas tributarias aplicadas desde el 2009 hasta diciembre 2015, para a partir de su aplicación analizar el desempeño de las importaciones. Los gravámenes arancelarios, el ICE, el IVA, salvaguardias

Palabras Claves: impuestos; salvaguardias; importaciones; sobretasas; tarifas arancelarias

Abstract: This research analysis the liquor sector which represents an important item of the Ecuadorian economy, it is composed of importers and local producers who in many cases need the imported goods for their production. All the states seek to improve their tax collection for the execution of works or improvement of the services delivered to the citizenship, whit this purpose, Ecuador Government applied a series of taxes as VAT value added tax, special consumption tax ICE , tariffs on imports, safeguards, imposed on IR income, among others. The present study focused on the import sector by the series of tax measures applied since 2009 to 2015, and analyze the performance of imports from its application. Tariffs, ICE, VAT, safeguards have reduced imports and affected the sale price of beverages and consumption in establishments.

Key Words: taxes; safeguards; imports; surcharges; tariff rates 


\section{Introducción}

El sector terciario de la economía que comprende, entre otras, las actividades de importación y comercialización de licores, constituye un insumo en otras áreas como la de servicios hoteleros y de diversión, crea un importante movimiento económico por los montos de transacciones y las fuentes de trabajo que genera.

En el país, como en otras naciones, se han impuesto una seria de tributos que buscan recaudación de impuestos y los establecidos al comercio exterior con la finalidad de imponer barreras que permitan regular las importaciones y exportaciones desde las decisiones gubernamentales.

Desde el año 2013 se han aplicado una serie de impuestos orientados a diversos productos y actividades, muchos de ellos han incluido al sector licorero, mismo que debe ajustarse tanto a normas de importación así como a gravámenes, que en el caso del ICE cada año es ajustado de acuerdo al índice de precios al consumidor, es también importante mencionar la obligatoriedad a partir del año 2015 del etiquetado fiscal en los envases de whisky, vodka, tequila y ron que ingresan al territorio nacional. (Servicio Nacional de Aduanas, 2015), y su ampliación a otros licores como vino, coñac, brandy, pisco, aguardiente, entre otros (excepto la cerveza), lo cual involucra además la generación de procedimientos administrativos.

La aplicación de los impuestos ha impactado en las importaciones y en el precio de los licores en el Ecuador desde el año 2013 hasta el 2105, año en el cual se aplicaron las salvaguardias, que debían ser eliminadas hasta junio del 2016 pero por motivos atribuidos a la crisis económica, se amplió su desmantelamiento hasta junio del 2017.

\section{Métodos}

La metodología para la presente investigación es de tipo cualitativa, en la misma se efectuó un enfoque exploratorio - descriptivo, se realizó un análisis de la literatura relacionada al tema propuesto, importaciones, aranceles, medidas tributarias, sector hotelero, y se analizó la influencia de estos en el consumo de licores. (Hernandez Sampieri, Fernendez Collado , \& Baptista Lucio, Metodología de la Investigación, 2014)

El análisis se realiza desde enero del 2012 hasta diciembre del 2015 para visualizar el comportamiento del mercado licorero con la aplicación de políticas comerciales e impuestos al sector, se ha realizado un levantamiento de datos de diversas fuentes oficiales como el Banco Central del Ecuador (BCE) y Servicio de Rentas Internas (SRI) Servicio Nacional de Aduanas (SENAE).

\section{Resultados}

El comercio internacional analiza las relaciones comerciales entre países, y promotores del libre comercio como Adam Smith y David Ricardo consideraron que ninguna nación es autosuficiente por sí misma. Esta visión del libre comercio y el proteccionismo, han generado diversas perspectivas y la adopción de medidas a nivel mundial. Según mencionan Krugman y 
Obstfeld (2006) desde la Segunda Guerra Mundial, las democracias avanzadas, han aplicado una política general consistente en suprimir las barreras al comercio internacional; esta política reflejaba la visión de que el libre comercio era una fuerza, no sólo de prosperidad, sino también para la paz mundial.

Sin embargo a nivel mundial se han implementado diversos instrumentos que permiten limitar los niveles de importación para una serie de productos; los niveles de restricción del intercambio comercial son un insumo esencial para negociadores y cualquier estudio para intentar comprender la institucionalidad y política aplicable a la protección comercial. (Kee, Nicita, \& Olarreaga, 2009)

Los estados aplican políticas económicas y comerciales con la finalidad de controlar los diferentes factores económicos del país así como los presupuestos estatales.

El investigador Lucas Pacheco, citado por Juan Paz y Miño (2015, pág. 25), dice al respecto de políticas económicas: "Se ha de entender por política económica a la intervención del Estado en la economía, de conformidad con estrategias derivadas de las relaciones de poder. Es una parte fundamental de las políticas públicas"

La recaudación de impuestos se remonta a épocas coloniales, en el siglo XVI nace uno de los primeros impuestos que estaba ligado a la actividad minera (Servicio de Rentas internas, 2012).

Al iniciar la vida como Estado (1830), en la cual se reguló fundamentalmente la "contribución de los indígenas" y las aduanas, desde ese año, los regímenes económicos y sociales han generado una serie de regulaciones y cambios en el área de impuestos, bajo esta perspectiva un hito que es importante mencionar es el de la Revolución Juliana en 1925, misma que se dio en respuesta a varias condiciones económicas históricas, por tanto el interés en el orden fiscal y tributario para solucionar problemas de hacienda pública. (Paz y Miño Cepeda, 2015, págs. 112-117).

El sector de los licores históricamente ha sido regulado, en el año 1795 se trató de ponerlo en vigencia con los estancos, que embargaban o prohibían el curso y venta libre de varias mercancías, entre ellas licor, tabaco, sal, explosivos y fósforos. En el gobierno Juliano centralizó totalmente los estancos de alcohol y tabaco, en 1930 la Legislatura expidió la Ley de Autonomía Económica que destinó a las Municipalidades del país 0,50 sucres, por cada litro de aguardiente que se consumiera en la respectiva jurisdicción, lo cual redujo los ingresos estatales haciendo que el Gobierno de Alfredo Baquerizo Moreno incremente el precio de venta de estos productos, lo que repercutió en un descenso aún mayor de las ventas legales y el crecimiento del contrabando. (Paz y Miño Cepeda, 2015, págs. 121-122)

En la actualidad las políticas comerciales se basan en siete instrumentos principales: aranceles, subsidios, cuotas de importación, limitación voluntaria de las exportaciones, requisitos de contenido local, políticas administrativas y políticas contra el dumping. (Hill, 2011) 
Los aranceles son impuestos aplicados a las importaciones y en ciertos casos a las exportaciones, y pueden ser fijos o ad-valorem, es uno de los instrumentos más simples empleados a nivel mundial, mismos que has buscado reducirse y regularse a partir del Acuerdo General de Aranceles y Comercio (GATT). Es importante considerar que el gobierno gana, porque los aranceles aumentan sus ingresos, los productores nacionales ganan, porque los aranceles les brindan protección contra competidores foráneos, pues aumentan el costo de las mercancías importadas y los consumidores pierden, porque tienen que pagar más por éstas. (Hill, 2011)

La mayoría de países en el mundo han reducido significativamente sus tarifas arancelarias en el marco de la GATT/WTO. Sin embrago, muchos países han creado nuevas barreras que incluyen leyes, políticas y prácticas para proteger sus ineficiencias internas o de manera artificial estimular las exportaciones. (Sarfati, 1998) (Islam, Mohamad-Ismail, \& Siwar, 2010)

El GATT en sus siglas en español es el Acuerdo General sobre Comercio y Aranceles, es un convenio en el cual se establecen las pautas de alcance comercial y concesiones arancelarias. (Perez-Capotos Contreras, 2003)

Todos los países imponen reglamentaciones técnicas acerca de embalajes, características y composición de los productos, etiquetado, entre otros. En el contexto del comercio internacional muchas de estas reglas pueden ser usadas como barreras para arancelarias. Varias de estas reglas violan las disposiciones de la Organización Mundial del Comercio (OMC) que solicita a los países miembros tratar a los productos importados en igualdad de condiciones que a la producción nacional. (Sumner, Smith, \& Rosson, 2015)

El Ecuador regula el establecimiento de medidas arancelarias cuya forma de expresión puede ser en términos advalorem, es decir un porcentaje sobre el valor en aduana, específico en términos monetarios sobre unidad de medida, o mixtos, a través del Código Orgánico de Producción, Comercio e Inversiones (COPCI), mismo que en los temas pertinentes al comercio exterior ajusta su legislación a la normativa de la OMC. (2010). Las medidas no arancelarias, en tanto se aplican para dar cumplimiento a lo dispuesto en tratados internacionales debidamente ratificados por el estado para corregir desequilibrios en la balanza de pagos.

El sector licorero desde tiempos remotos, ha sido objeto de la aplicación de medidas tributarias para reducir el contrabando y contribuir a generar recursos para el Estado.

Los productos comprendidos en el capítulo 22 del arancel de aduanas, y particularmente los licores, desde el año 2013 ha venido registrando una serie de impuestos, tasas y requerimientos administrativos que hacen que especialmente los productos importados deban someterse a procedimientos especiales, que hacen incrementar su precio al consumidor final; así desde el mes abril de 2015, mediante Resolución Nro. SENAE-DGN-2015-0185-RE se estableció la obligatoriedad de etiquetar todas las botellas importadas de whisky, ron, tequila y vodka, como medida de seguridad que permitirá la identificación, registró la trazabilidad de la mercancía. (Servicio Nacional de Aduanas, 2015) 
De igual manera el impuesto a los consumos especiales ICE, aplicado sobre ciertos bienes y servicios sean nacionales o importados, que en el caso de los licores se grava en función de los litros de alcohol puro en las bebidas.

En el mes de marzo del 2015 el Comité de Comercio Exterior del Ecuador en función de sus atribuciones contempladas en el Código Orgánico de Producción, Comercio e Inversiones (2010), expidió la resolución No. 11 - 2015 (2015) que afectó a aproximadamente 2700 subpartidas es decir un 30\% del total del universo arancelario. Las bebidas alcohólicas que se vieron afectadas por esta medida fueron principalmente la cerveza de malta, vinos, vodka, tequila y aguardiente, así como concentrados para la elaboración de whisky, ron y otros aguardientes.

Para el presente estudio se han considerado las siguientes partidas: 2203.00 .00 cerveza de malta, 2204.29.90 vinos, 2208.30.00.90 whisky, 2208400090 ron, 2208600000 vodka, 2208902000 Tequila.

Si bien los aranceles a la importación se han mantenido estables, las salvaguardias, y el ICE con una tarifa advalorem y específica que se ajusta anual y acumulativamente en función de la variación anual del índice de precios al consumidor (IPC) a noviembre de cada año, y aplicable desde el mes de enero del siguiente año. (Ley Orgánica de Régimen Tributario Interno, 2011), hace que la carga tributaria sea cada vez mayor.

Tabla 1. Tabla de Aranceles e impuestos aplicados a los licores

\begin{tabular}{|c|c|c|c|c|c|c|c|c|c|}
\hline \multirow[b]{2}{*}{ Impuesto } & \multirow[b]{2}{*}{ Partidas } & \multicolumn{2}{|c|}{2012} & \multicolumn{2}{|c|}{2013} & \multicolumn{2}{|c|}{2014} & \multicolumn{2}{|c|}{2015} \\
\hline & & Fijo & $\begin{array}{l}\text { Ad- } \\
\text { val. }\end{array}$ & Fijo & $\begin{array}{l}\text { Ad- } \\
\text { val. }\end{array}$ & Fijo & $\begin{array}{l}\text { Ad- } \\
\text { val. }\end{array}$ & Fijo & $\begin{array}{l}\text { Ad- } \\
\text { val. }\end{array}$ \\
\hline \multirow{6}{*}{ Arancel } & 2203.00 .00 & $\begin{array}{r}\text { USD } \\
0.25 \\
\text { G.A.L. }\end{array}$ & $1 \%$ & $\begin{array}{r}\text { USD } \\
0.25 \\
\text { G.A.L. }\end{array}$ & $1 \%$ & $\begin{array}{r}\text { USD } \\
0.25 \\
\text { G.A.L. }\end{array}$ & $1 \%$ & $\begin{array}{r}\text { USD } \\
0.25 \\
\text { G.A.L. }\end{array}$ & $1 \%$ \\
\hline & 2204.29 .90 & & $30 \%$ & & $30 \%$ & & $30 \%$ & & $30 \%$ \\
\hline & 2208.30 .00 .90 & $\begin{array}{r}\text { USD } \\
0.25 \\
\text { G.A.L. }\end{array}$ & $1 \%$ & $\begin{array}{r}\text { USD } \\
0.25 \\
\text { G.A.L. }\end{array}$ & $1 \%$ & $\begin{array}{r}\text { USD } \\
0.25 \\
\text { G.A.L. }\end{array}$ & $1 \%$ & $\begin{array}{r}\text { USD } \\
0.25 \\
\text { G.A.L. }\end{array}$ & $1 \%$ \\
\hline & 2208400090 & $\begin{array}{r}\text { USD } \\
0.25 \\
\text { G.A.L. }\end{array}$ & $1 \%$ & $\begin{array}{r}\text { USD } \\
0.25 \\
\text { G.A.L. }\end{array}$ & $1 \%$ & $\begin{array}{r}\text { USD } \\
0.25 \\
\text { G.A.L. }\end{array}$ & $1 \%$ & $\begin{array}{r}\text { USD } \\
0.25 \\
\text { G.A.L. }\end{array}$ & $1 \%$ \\
\hline & 2208600000 & $\begin{array}{r}\text { USD } \\
0.25 \\
\text { G.A.L. }\end{array}$ & $1 \%$ & $\begin{array}{r}\text { USD } \\
0.25 \\
\text { G.A.L. }\end{array}$ & $1 \%$ & $\begin{array}{r}\text { USD } \\
0.25 \\
\text { G.A.L. }\end{array}$ & $1 \%$ & $\begin{array}{r}\text { USD } \\
0.25 \\
\text { G.A.L. }\end{array}$ & $1 \%$ \\
\hline & 2208902000 & $\begin{array}{r}\text { USD } \\
0.25 \\
\text { G.A.L. }\end{array}$ & $1 \%$ & $\begin{array}{r}\text { USD } \\
0.25 \\
\text { G.A.L. }\end{array}$ & $1 \%$ & $\begin{array}{r}\text { USD } \\
0.25 \\
\text { G.A.L. }\end{array}$ & $1 \%$ & $\begin{array}{r}\text { USD } \\
0.25 \\
\text { G.A.L. }\end{array}$ & $1 \%$ \\
\hline \multirow{6}{*}{ Salvaguardia } & 2203.00 .00 & & & & & & & & $25 \%$ \\
\hline & 2204.29 .90 & & & & & & & & $45 \%$ \\
\hline & 2208.30 .00 .90 & & & & & & & & $25 \%$ \\
\hline & 2208400090 & & & & & & & & $25 \%$ \\
\hline & 2208600000 & & & & & & & & $25 \%$ \\
\hline & 2208902000 & & & & & & & & $25 \%$ \\
\hline IVA & & & $12 \%$ & & $12 \%$ & & $12 \%$ & & $12 \%$ \\
\hline ICE* & & 6.20 & $75 \%$ & 6.93 & $75 \%$ & 6.93 & $75 \%$ & 7.10 & $75 \%$ \\
\hline
\end{tabular}

Fuente: Servicio de Rentas Internas, Resoluciones Comité de comercio Exterior

El impacto de la aplicación de los gravámenes puede presentarse en diferentes aspectos, el incremento en el precio final, el nivel de importaciones y el nivel de recaudaciones entre otros. 
En cuanto al precio, para analizar el impacto se establecen supuestos para cada partida y se suman todos los gravámenes para establecer el valor final de los productos importados, de este ejercicio, se desprende que las variaciones entre el año 2012 y el 2015 manteniendo una base imponible estable para los cuatro años analizados, que el precio ha ido incrementando.

Tabla 2. Cálculos de Impuestos aplicados a la importación de licores

\begin{tabular}{|c|c|c|c|c|c|c|c|c|c|c|c|}
\hline \multirow[b]{2}{*}{ Bebida } & \multirow[b]{2}{*}{$\begin{array}{c}\text { Base } \\
\text { Imponible* }^{*}\end{array}$} & \multirow[b]{2}{*}{$\begin{array}{c}\text { G.A. } \\
\text { L }\end{array}$} & \multicolumn{2}{|c|}{$\begin{array}{c}\text { Derechos } \\
\text { aduaneros }\end{array}$} & \multirow[t]{2}{*}{ Salvag. } & \multirow[t]{2}{*}{$\begin{array}{c}\text { Fodinfa } \\
\mathbf{0 , 5 \%}\end{array}$} & \multirow[t]{2}{*}{$\begin{array}{l}\text { Valor ex } \\
\text { aduana }\end{array}$} & \multicolumn{2}{|c|}{ ICE } & \multirow[t]{2}{*}{ IVA } & \multirow[t]{2}{*}{ TOTAL } \\
\hline & & & Fijo & $\begin{array}{l}\text { Ad } \\
\text { val. }\end{array}$ & & & & Fijo & $\begin{array}{l}\text { Ad } \\
\text { val. }\end{array}$ & & \\
\hline \multicolumn{2}{|c|}{ Cerveza de Malta } & $4 \%$ & & & & & & & & & \\
\hline 2012 & 1,65 & & 0,01 & 0,02 & & 0,01 & 1,68 & 0,25 & 1,26 & 0,38 & 3,58 \\
\hline 2013 & 1,65 & & 0,01 & 0,02 & & 0,01 & 1,68 & 0,28 & 1,26 & 0,39 & 3,61 \\
\hline 2014 & 1,65 & & 0,01 & 0,02 & & 0,01 & 1,68 & 0,28 & 1,26 & 0,39 & 3,61 \\
\hline 2015 & 1,65 & & 0,01 & 0,02 & 0,41 & 0,01 & 2,10 & 0,28 & 1,57 & 0,47 & 4,43 \\
\hline Vino & & $12 \%$ & & & & & & & & & \\
\hline 2012 & 6,75 & & 0 & 2,03 & & 0,03 & 8,81 & 0,74 & 6,61 & 1,94 & 18,10 \\
\hline 2013 & 6,75 & & 0 & 2,03 & & 0,03 & 8,81 & 0,83 & 6,61 & 1,95 & 18,20 \\
\hline 2014 & 6,75 & & 0 & 2,03 & & 0,03 & 8,81 & 0,83 & 6,61 & 1,95 & 18,20 \\
\hline 2015 & 6,75 & & 0 & 2,03 & 1,69 & 0,03 & 10,50 & 0,85 & 7,87 & 2,31 & 21,53 \\
\hline Ron & & $40 \%$ & & & & & & & & & \\
\hline 2012 & 7,80 & & 0,10 & 0,08 & & 0,04 & 8,02 & 2,48 & 6,01 & 1,98 & 18,49 \\
\hline 2013 & 7,80 & & 0,10 & 0,08 & & 0,04 & 8,02 & 2,77 & 6,01 & 2,02 & 18,82 \\
\hline 2014 & 7,80 & & 0,10 & 0,08 & & 0,04 & 8,02 & 2,77 & 6,01 & 2,02 & 18,82 \\
\hline 2015 & 7,80 & & 0,10 & 0,08 & 1,95 & 0,04 & 9,97 & 2,84 & 7,48 & 2,43 & 22,97 \\
\hline Wishky & & $45 \%$ & & & & & & & & & \\
\hline 2012 & 9,15 & & 0,11 & 0,09 & & 0,05 & 9,40 & 2,79 & 7,05 & 2,31 & 21,55 \\
\hline 2013 & 9,15 & & 0,11 & 0,09 & & 0,05 & 9,40 & 3,12 & 7,05 & 2,35 & 21,92 \\
\hline 2014 & 9,15 & & 0,11 & 0,09 & & 0,05 & 9,40 & 3,12 & 7,05 & 2,35 & 21,92 \\
\hline 2015 & 9,15 & & 0,11 & 0,09 & 2,29 & 0,05 & 11,69 & 3,20 & 8,77 & 2,84 & 26,74 \\
\hline Vodka & & $28 \%$ & & & & & & & & & \\
\hline 2012 & 6,50 & & 0,07 & 0,07 & & 0,03 & 6,67 & 1,74 & 5,00 & 1,61 & 15,01 \\
\hline 2013 & 6,50 & & 0,07 & 0,07 & & 0,03 & 6,67 & 1,94 & 5,00 & 1,63 & 15,24 \\
\hline 2014 & 6,50 & & 0,07 & 0,07 & & 0,03 & 6,67 & 1,94 & 5,00 & 1,63 & 15,24 \\
\hline 2015 & 6,50 & & 0,07 & 0,07 & 1,63 & 0,03 & 8,29 & 1,96 & 6,22 & 1,98 & 18,70 \\
\hline Tequila & & $30 \%$ & & & & & & & & & \\
\hline 2012 & 5,50 & & 0,08 & 0,06 & & 0,03 & 5,66 & 1,86 & 4,24 & 1,41 & 13,17 \\
\hline 2013 & 5,50 & & 0,08 & 0,06 & & 0,03 & 5,66 & 2,08 & 4,24 & 1,44 & 13,42 \\
\hline 2014 & 5,50 & & 0,08 & 0,06 & & 0,03 & 5,66 & 2,08 & 4,24 & 1,44 & 13,42 \\
\hline 2015 & 5,50 & & 0,08 & 0,06 & 1,38 & 0,03 & 7,03 & 2,10 & 5,27 & 1,73 & 16,39 \\
\hline ijo li & ro sin consider & rar in & ne & & & & & & & & \\
\hline & & & & & & & & Fuen & te: $S$ & $\mathrm{I}, \mathrm{CON}$ & EX, Autora \\
\hline
\end{tabular}


Tabla 3. Valores de licores luego de impuestos

Valores en USD

\begin{tabular}{|l|r|r|r|r|r|}
\hline & $\begin{array}{c}\text { Base } \\
\text { Imponible }\end{array}$ & $\mathbf{2 0 1 2}$ & $\mathbf{2 0 1 3}$ & $\mathbf{2 0 1 4}$ & $\mathbf{2 0 1 5}$ \\
\hline Cerveza & 1,65 & 3,58 & 3,61 & 3,61 & 4,43 \\
\hline Vino & 6,75 & 18,10 & 18,20 & 18,20 & 21,53 \\
\hline Ron & 7,80 & 18,49 & 18,82 & 18,82 & 22,97 \\
\hline Wishky & 9,15 & 21,55 & 21,92 & 21,92 & 26,74 \\
\hline Vodka & 6,50 & 15,01 & 15,24 & 15,24 & 18,70 \\
\hline Tequila & 5,50 & 13,17 & 13,42 & 13,42 & 16,39 \\
\hline
\end{tabular}

Fuente: Tabla 2

Los licores más afectados son los que tienen mayor grado alcohólico, como whisky, ron y vodka, sin embargo el vino al ser una bebida de menor contenido de alcohol, la tarifa arancelaria del 30\% advalorem. En el 2015, año en el que además se agregó el valor de la etiqueta fiscal 0,25 usd por botella se visualiza el incremento más fuerte.

Cada año el impacto de los impuestos en las bebidas alcohólicas llega incluso a triplicar el valor de la base imponible establecida para la aplicación de gravámenes. Afectando los precios y reduciendo su consumo y por tanto las importaciones, lo cual es el objetivo del Estado para mitigar el impacto de la crisis de balanza de pagos.

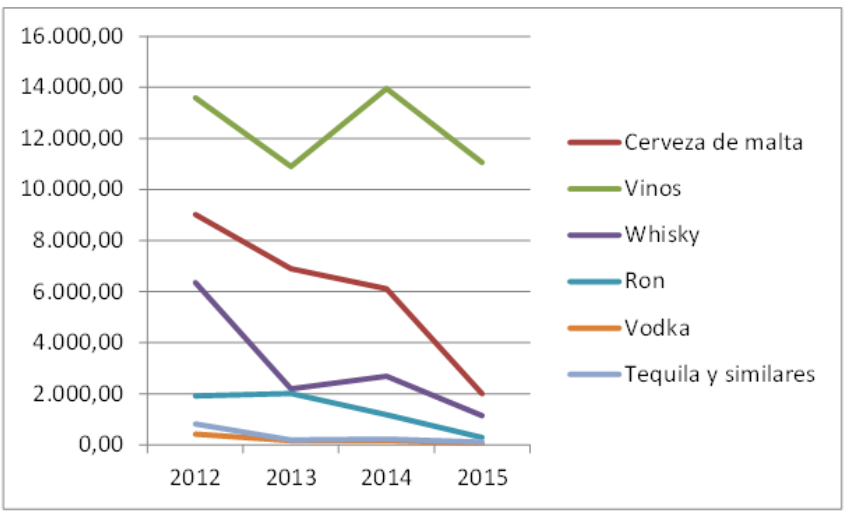

Figura 1. Tendencia de importaciones de licores Fuente: Banco Central del Ecuador

Como se puede evidenciar, los diferentes tipos de licores han sufrido un importante decremento en el nivel de importaciones, lo que ha generado también una menor recaudación de tributos.

Entre el año 2012 al 2015 en promedio se evidencia un 54\% de reducción de las importaciones de licores, siendo el menos afectado el sector vinícola.

El análisis de la reducción en la recaudación tributaria, muestra también la misma tendencia, si bien la estadística que se presenta a continuación no sólo analiza sólo el ICE de licores se puede observar la tendencia en el periodo analizado, llegando a un decremento del $24 \%$ de recaudación, lo cual reduce el ingreso del estado para el gasto social. 


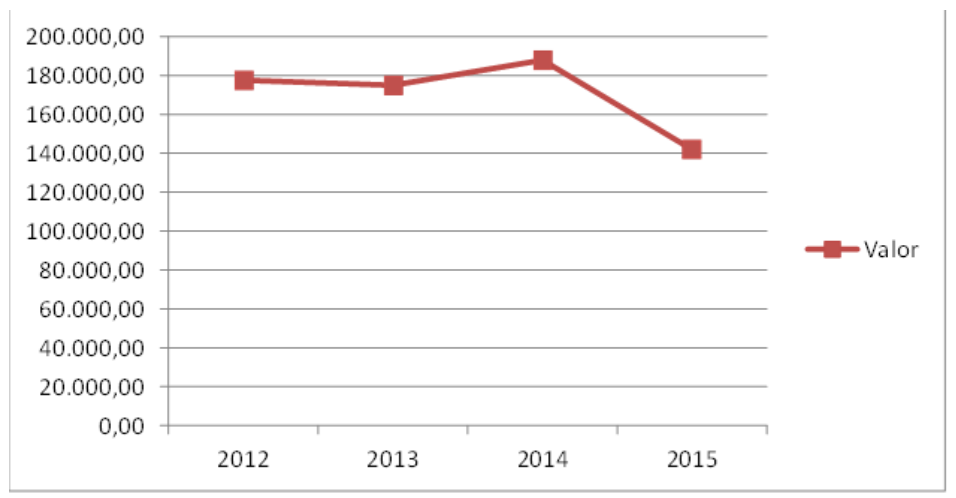

Figura 2. Recaudación ICE Importaciones

Fuente: Servicio de Rentas Internas, 2016

Tabla 4. Ice Importaciones

\begin{tabular}{|l|l|r|}
\hline Año & Valor & \% Variación \\
\hline 2012 & $177.516,26$ & $-1,46 \%$ \\
\hline 2013 & $174.931,52$ & $7 \%$ \\
\hline 2014 & $187.839,49$ & $-24,42 \%$ \\
\hline 2015 & $141.976,00$ & \\
\hline
\end{tabular}

Fuente: Servicio de Rentas internas

En el Ecuador a más de los aranceles a las bebidas alcohólicas, las regulaciones existentes para la venta este tipo de bebidas que en su momento efectúo el Ministerio de Turismo en establecimientos del sistema turísticos (restaurantes, bares cafeterías, salas de banquetes y hoteles), afectó la comercialización

\section{Conclusiones}

El Estado considera como su principal fuente de ingresos la aplicación del impuestos, y el sector licorero ha sido históricamente afectado con la aplicación de gravámenes, éste sector no sólo genera empleo por sí mismo en el proceso de comercialización, sino que además es generador de empleo a través de actividades enfocadas a la diversión e incluso el turismo, del análisis se desprende que las diferentes medidas aplicadas han afectado de manera importante a este segmento, en el presente análisis se ha considerado tres aspectos, el nivel de precios, el nivel de recaudación del ICE y el nivel de importaciones, y se considera que los tres aspectos muestran importantes reducciones especialmente en el último año.

En el país por tradición al ser productor agrícola en el cual se cuenta con cultivos de caña de azúcar se ha elaborado de manera artesanal especialmente aguardiente que por sus características identifican a las zonas donde se producen. Un alternativa para minimizar este impacto y generar fuentes de empleo además de considerar la posibilidad de exportar sería el impulso a la industrialización de estos productos, que por no contar con los permisos necesarios han debido en algunos casos cambiar su producción.

Es importante realizar un análisis de la incidencia que tuvo los aranceles en el consumo de licores, principalmente en alimentos y bebidas. 


\section{Bibliografía}

Asamblea Nacional. (2011). Ley Orgánica de Régimen Tributario Interno. Registro Oficial. Quito, Pichincha: Registro Oficial.

Asamblea Nacional. (Marzo de 2015). Resolución No. 11 - 2015. Quito: Registro Oficial.

Asamblea Nacional del Ecuador. (29 de Diciembre de 2010). Código Orgánico de Producción, Comercio en Inversiones. Registro Oficial. Quito, Pichincha, Ecuador: Registro Oficial.

Banco Central del Ecuador. (Diciembre de 2012; 2013; 2014; 2015). Banco Central del Ecuador. $\begin{array}{llllll}\text { Recuperado el junio de } 6 \text { de } & \text { de }\end{array}$ https://www.bce.fin.ec/comercioExteriorBi/comercio/consultaTotXNandinaConGraficoV 2.jsp

Hernandez Sampieri, R., Fernandez Collado, C., \& Baptista Lucio, M. (2014). Metodología de la Investigación. México: McGraw-Hill.

Hill, C. (2011). Negocios Internacionales . México: Mc Graw Hill.

Islam, R., Mohamad-Ismail, S., \& Siwar, C. (2010). Science Publications. Obtenido de American Journal of Environmental Sciences: http://thescipub.com/PDF/ajessp.2010.95.102.pdf

Kee, H. L., Nicita, A., \& Olarreaga, M. (2009). World Bank Group S.A. Obtenido de http://siteresources.worldbank.org/INTRES/Resources/4692321107449512766/ecoj_2209.pdf

Paul R. Krugman, M. O. (2006). Economía Internacional, teoría y política. Madrid: Perason.

Paz y Miño Cepeda, J. (2015). Historia de los Impuestos en el Ecuador. Quito: SRI- PUCE_THE.

Perez-Capotos Contreras, J. (2003). Los subsidios gubernamentales como prácticas desleales del comercio internacional. Revista de Derecho de la Pontificia Universidad Católica de Valparaiso, 403-422.

Sarfati, G. (Mayo de 1998). European Integration online Papers. Recuperado el 2016, de http://eiop.or.at/eiop/texte/1998-002.htm

Servicio de Rentas internas. (2012). Una nueva política fiscal para el Buen Vivir: La equidad como soporte del pacto fiscal. Quito: Abya-Yala.

Servicio de Rentas Internas. (Diciembre de 2013). Servicio de Rentas Internas. Recuperado el 6 de junio de 2016, de http://www.sri.gob.ec/web/guest/estadisticas-generales-derecaudacion?p_auth=q15CqwEA\&p_p_id=busquedaEstadisticas_WAR_BibliotecaPortle t_INSTANCE_EVo6\&p_p_lifecycle=1\&p_p_state=normal\&p_p_mode=view\&p_p_col_ id=column-1\&p_p_col_count=2\&_busquedaEstadisticas_

Servicio Nacional de Aduanas. (abril de 2015). Resolución SENAE-DNG-2015-0185-RE. Resolución. Guayaquil, Guayas, Ecuador.

Sumner, D. A., Smith, V. H., \& Rosson, C. P. (2015). Cite Seer X. Obtenido de http://citeseerx.ist.psu.edu/viewdoc/download;jsessionid=3951FB337EFCA797B984426 7E3F279BF?doi=10.1.1.553.8355\&rep=rep1\&type=pdf 